\title{
Comparing the accuracy of the three popular clinical dehydration scales in children with diarrhea
}

\author{
Kimberly Pringle ${ }^{1,6^{*}}$, Sachita P Shah ${ }^{1}$, Irenee Umulisa ${ }^{2}$, Richard B Mark Munyaneza ${ }^{2}$, Jean Marie Dushimiyimana ${ }^{3}$, \\ Katrina Stegmann ${ }^{3}$, Juvenal Musavuli ${ }^{4}$, Protegene Ngabitsinze ${ }^{4}$, Sara Stulac ${ }^{5}$ and Adam C Levine ${ }^{1,5}$
}

\begin{abstract}
Background: Dehydration due to acute gastroenteritis is one of the leading causes of mortality in children worldwide. The World Health Organization (WHO) scale, the Gorelick scale, and the Clinical Dehydration Scale (CDS) were created to estimate percentage dehydration in children with gastroenteritis based on clinical signs. Of these, only the CDS has been prospectively validated against a valid gold standard, though never in low- and middleincome countries. The purpose of this study is to determine whether these clinical scales can accurately assess dehydration status in children when performed by nurses or general physicians in a low-income country.

Methods: We prospectively enrolled a non-consecutive sample of children presenting to three Rwandan hospitals with diarrhea and/or vomiting. A health care provider documented clinical signs on arrival and weighed the patient using a standard scale. Once admitted, the patient received rehydration according to standard hospital protocol and was weighed again at hospital discharge. Receiver operating characteristic (ROC) curves were created for each of the three scales compared to the gold standard, percent weight change with rehydration. Sensitivity, specificity, and likelihood ratios were calculated based on the best cutoff points of the ROC curves.
\end{abstract}

Results: We enrolled 73 children, and 49 children met eligibility criteria. Based on our gold standard, the children had a mean percent dehydration of 5\% on arrival. The WHO scale, Gorelick scale, and CDS did not have an area under the ROC curve statistically different from the reference line. The WHO scale had sensitivities of 79\% and 50\% and specificities of $43 \%$ and $61 \%$ for severe and moderate dehydration, respectively; the 4- and 10-point Gorelick scale had sensitivities of $64 \%$ and $21 \%$ and specificities of $69 \%$ and $89 \%$, respectively, for severe dehydration, while the same scales had sensitivities of $68 \%$ and $82 \%$ and specificities of $41 \%$ and $35 \%$ for moderate dehydration; the CDS had a sensitivity of $68 \%$ and specificity of $45 \%$ for moderate dehydration.

Conclusion: In this sample of children, the WHO scale, Gorelick scale, and CDS did not provide an accurate assessment of dehydration status when used by general physicians and nurses in a developing world setting.

\section{Background}

Diarrhea has the highest incidence of any childhood disease in all regions of the world and kills approximately 1.9 million children each year, accounting for $19 \%$ of all deaths in children under 5 [1,2]. The World Health Organization (WHO), the Centers for Disease Control and Prevention (CDC), the American Academy of Pediatrics (AAP), and the European Society of Pediatric Gastroenterology and Nutrition (ESPGAN) all support rehydration with oral rehydration solution (ORS) for children with

\footnotetext{
* Correspondence: Kimberly.pringle@brown.edu

'Department of Emergency Medicine, Brown University Alpert Medical

School, Providence, RI, USA

Full list of author information is available at the end of the article
}

mild to moderate dehydration, reserving intravenous (IV) fluids for children with severe dehydration [3-6]. Oral rehydration solution is a hypo-osmolar solution composed of salts, sugar, and citrate, while the recommended intravenous fluids include lactated Ringer's or normal saline [4,6-8].

In order to apply the most appropriate treatment for dehydration in children with gastroenteritis, healthcare providers must first accurately assess the severity of dehydration [5]. Underestimating fluid deficit, and not providing proper rehydration with either ORS or IV therapy can lead to acidosis, electrolyte disturbances, acute kidney injury, or even death. Alternatively, overestimating fluid deficit can lead to unnecessary interventions,

\section{实 Springer}

(C) 2011 Pringle et al; licensee Springer. This is an Open Access article distributed under the terms of the Creative Commons Attribution License (http://creativecommons.org/licenses/by/2.0), which permits unrestricted use, distribution, and reproduction in any medium, provided the original work is properly cited. 
longer hospital stays, and increased adverse events in children $[9,10]$. Accurate fluid assessment is of utmost importance in low- and middle-income countries, where many patients travel several hours to reach a healthcare facility, and resources such as IV fluids and hospital beds are scarce.

Several organizations and research institutions have developed scales to estimate dehydration status using clinical signs. The most popular are the WHO scale, the Gorelick scale (created at the Children's Hospital of Philadelphia), and the Clinical Dehydration Scale (CDS; created at the Hospital for Sick Children in Toronto) (please see Tables 1, 2, and 3 for scales). The scales predict percent dehydration for slightly different age groups; the CDS is for children between 1 month and 3 years, while the other two scales are for children between 1 month and 5 years. The WHO scale classifies children by grouping severity of symptoms, while the CDS has a scoring system. The Gorelick scale uses binary categorization of symptoms either as no dehydration or moderate/severe dehydration, and severity is determined by the number of physical signs present. Each scale also predicts a slightly different range for percent volume loss.

Thus far, only the CDS has been prospectively validated against the accepted gold standard of percent weight change with rehydration at a single pediatric referral center in North America [11]. None of these scales have been validated in low- or middle-income countries where disease patterns may differ from highincome countries, patients often present later in the course of their disease, and healthcare providers often lack specialty training. The purpose of this study was to determine the accuracy of the WHO scale, Gorelick scale, and CDS in a resource-limited setting.

\section{Methods}

\section{Study design}

In this study we enrolled a non-consecutive cohort of children presenting with symptoms of diarrhea and/or vomiting to one of three district hospitals in Rwanda. The study was approved by both the Partners Healthcare (Massachusetts General Hospital) Institutional Review Board and the Rwanda National Ethics Committee. The child's parent or guardian provided either written on verbal consent in the local language, Kinyarwanda.

\section{Study setting and population}

All three district hospitals, Kirehe, Rwinkwavu, and Butaro, serve rural and relatively impoverished populations. We enrolled children less than 15 years of age, the upper limit for admission to the pediatrics ward at each study hospital, but limited our analysis to children fitting within the predefined age ranges for each of the clinical dehydration scales. Each hospital has 25-40 inpatient pediatric beds and serves an estimated catchment area of 150,000 to 350,000 people. Enrollment occurred MarchJuly 2009 , and included all pediatric patients presenting with diarrhea and/or vomiting on weekdays from 7:00 a. m. - 5:00 p.m., and occasional nights and weekends based on availability of study staff.

\section{Data collection and methods of measurement}

When an eligible patient presented to one of the three district hospitals, the nurse or physician caring for the patient contacted the local study coordinator. The coordinator then explained the study and obtained written or verbal consent in Kinyarwanda. The child was then weighed on a standard scale. The physician or nurse admitting the patient noted demographic information, nutritional status, and the signs and symptoms of dehydration.

Once admitted, the patient was treated according to standard hospital protocols, based largely on WHO protocols for management of dehydration in children. After they underwent rehydration therapy, the patient was weighed again on the same scale, and a discharge weight was recorded.

\section{Data analysis}

First, basic descriptive statistics were calculated for our study population. We then took each of the clinical scales - WHO, Gorelick, and CDS - and classified each patient according to the scale. Receiver operating characteristic (ROC) curves were created for each of the three scales compared to the gold standard, percent weight change with rehydration. Percent weight change with rehydration was calculated by (rehydration weight - admission weight)/rehydration weight $\times 100 \%$. Sensitivity, specificity,

Table 1 WHO Scale for dehydration for children 1 month-5 years old

\begin{tabular}{cccc}
\hline & A & B & C \\
\hline Look at condition & Well, alert & Restless, irritable & Lethargic or unconscious \\
Eyes & Normal & Sunken & Sunken \\
Thirst & Drinks normally, not thirsty & Thirsty, drinks eagerly & Drinks poorly or not able to drink \\
Feel: Skin pinch & Goes back quickly & Goes back slowly & Goes back very slowly \\
\hline
\end{tabular}

Scoring: Fewer than two signs from column B and C: no signs of dehydration < 5\%, $\geq 2$ signs in column B: Moderate dehydration 5 - $10 \%, \geq 2$ signs in column C: $>$ $10 \%$ severe dehydration 
Table 2 The 10- and 4-point Gorelick Scale for dehydration: for children 1 month-5 years; 4-point scale physical exam signs highlighted in italic font

\begin{tabular}{ccc}
\hline Characteristic & No or minimal dehydration & Moderate to severe dehydration \\
\hline General appearance & Alert & Restless, lethargic, unconscious \\
Capillary refill & Normal & Prolonged or minimal \\
Tears & Present & Absent \\
Mucous membranes & Moist & Dry, very dry \\
Eyes & Normal & Sunken; deeply sunken \\
Breathing & Present & Deep; deep and rapid \\
Quality of pulses & Normal & Thready; weak or impalpable \\
Skin elasticity & Instant recoil & Recoil slowly; recoil $>2 \mathrm{~s}$ \\
Heart rate & Normal & Tachycardia \\
Urine output & Normal & Reduced; not passed in many hours
\end{tabular}

Scoring: 4 point scale (italics): $\geq 2$ Clinical Signs ( $4 \mathrm{pt}) \geq 5 \% \mathrm{BW} \Delta ;$

3 Clinical Signs ( $4 \mathrm{pt}) \geq 10 \% \mathrm{BW} \Delta ; 10$ point scale (all signs/symptoms):

$\geq 3$ Clinical Signs $\geq 5 \%$ BW $\Delta ; \geq 7$ Clinical Signs $\geq 10 \%$ BW $\Delta$

and likelihood ratios were calculated based on the best cutoff points for each of the ROC curves.

For the WHO and Gorelick scales, separate ROC curves were created for severe and moderate dehydration. For the CDS, ROC curves and sensitivities and specificities were created only for those children classified as having moderate dehydration ( $\geq 6 \%$ dehydration). All statistical analyses were performed using SPSS 16.0 (SPSS Inc., Chicago, IL).

\section{Results}

Seventy-three children were enrolled in the study. Two children died prior to discharge, 12 children had evidence of severe malnutrition, and 7 children were missing discharge weights, leaving 52 children for analysis. Of these 52 children, 49 children were between 1 month and 5 years old and could be classified by the Gorelick and WHO scale, while 48 children were between 1 month and 3 years old and could be classified by the CDS.

The mean age was 10.5 months for children 1-36 months and 11.14 months for children 1 month- 5 years. The median percent weight change for children between 1 and 36 months and children between 1 and 60 months was $4.8 \%$. Twenty-nine percent of children presented with severe dehydration, classified as > 10\% weight change between admission and discharge. The median length of stay was 4 days, with $88 \%$ of patients staying at least 3 days for both age groups.
None of the scales had an area under the ROC curve statistically different from the reference line (see Figures 1 , 2, 3, 4 and 5). Stratifying by healthcare provider (doctor versus nurse) did not improve the accuracy of any of the ROC curves (data not shown). The WHO scale for moderate dehydration (5-10\% percent weight change) had an area under the curve of $0.58(95 \% \mathrm{CI}=0.39-0.78)$; sensitivity was $50 \%$ and specificity $61 \%$; LR+ was 1.28 and LR0.82 . For severe dehydration ( $>10 \%$ percent body weight change) the WHO scale had an area under the curve of 0.58 ( $95 \% \mathrm{CI}=0.41-0.75)$; sensitivity was $79 \%$ and specificity 43\%; LR+ was 1.38 and LR- 0.50 . For moderate dehydration (between $5-10 \%$ percent body weight change) the 4- and 10-point Gorelick scales had an area under the curve of $0.62(95 \% \mathrm{CI}=0.45-0.78)$ and $0.58(95 \% \mathrm{CI}=$ $0.42-0.74$ ), respectively; sensitivities were $64 \%$ and $21 \%$, and specificities were $69 \%$ and $82 \%$, respectively; $L R+$ was 1.15 and 1.25 and LR- 0.78 and 0.53 , respectively. For severe dehydration ( $\geq 10 \%$ body weight change) the 4 - and 10-point Gorelick scale had areas under the curve of 0.62 (95\% CI $=0.45-0.79)$ and $0.60(95 \% \mathrm{CI}=0.44-0.77)$, respectively; sensitivities were $68 \%$ and $82 \%$, and specificities were $41 \%$ and $35 \%$, respectively; $L R+$ was 1.09 and 2.04, and LR- 0.52 and 0.88 , respectively. For the prediction of moderate dehydration (percent weight change $\geq 6 \%$ ), the CDS had an area under the curve of 0.64 (95\% $\mathrm{CI}=0.44-0.77)$. The sensitivity of the scale was $68 \%$ and the specificity $45 \%$, with a LR+ of 1.24 and LR- of 0.70 .

Table 3 CDS scale clinical features for prediction dehydration in children 1-36 months

\begin{tabular}{cccc}
\hline Characteristic & $\mathbf{0}$ & $\mathbf{1}$ & $\mathbf{2}$ \\
\hline General appearance & Normal & Thirsty, restless, or lethargic, but irritable when touched & Drowsy, limp, cold, sweaty, and/or comatose \\
Eyes & Normal & Slightly sunken & Very sunken \\
Mucous membranes & Moist & "Sticky" & Dry \\
Tears & Tears & Decreased tears & Absent tears \\
\hline
\end{tabular}

Scoring: 0: no dehydration $<3 \%, 1-4$ : some dehydration $\leq 3 \times<6 \%, 5-8$ : moderate dehydration $\geq 6 \%$ 


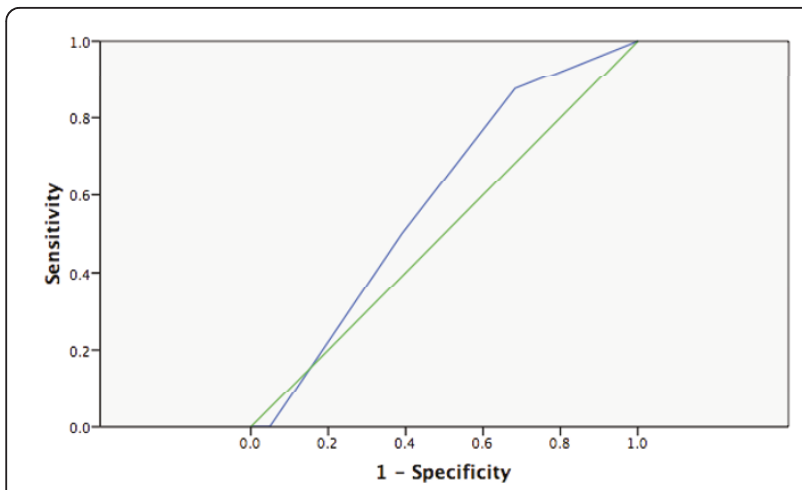

Figure 1 WHO scale predicting moderate (5-10\%) body weight change.

\section{Discussion}

While most experts agree that children with diarrhea should be treated based on the severity of their dehydration, with children receiving intravenous fluids for severe dehydration and oral rehydration solution for mild to moderate dehydration, there is no clear consensus on how best to determine the severity of dehydration, especially in resource-limited settings. The gold standard for dehydration is percent volume loss with diarrhea, which is defined as the difference between preillness weight and acute-illness weight divided by pre-illness weight. Because pre-illness weight from a pediatrician's office is often not available, especially in low- and middle-income countries, it is ideally substituted by stable post-rehydration weight or the child's weight after undergoing therapy. In fact, Gorelick et al. validated post-rehydration weight as a surrogate for pre-illness weight by demonstrating near perfect correlation $(r=$ 0.9988) between the two values in a small cohort of children with diarrhea [12]. In our study, we were not able to guarantee that participants had reached a stable post-rehydration weight prior to discharge. However, most children in the study by Gorelick et al. achieved a

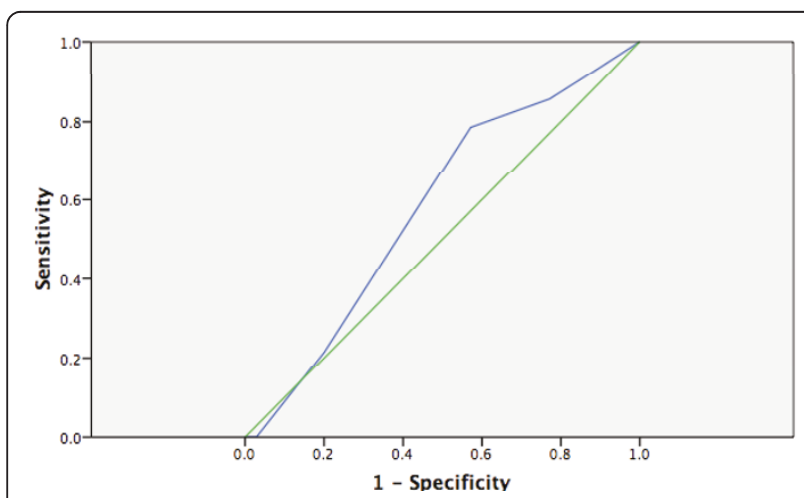

Figure 2 WHO scale predicting severe (> 10\%) body weight change.

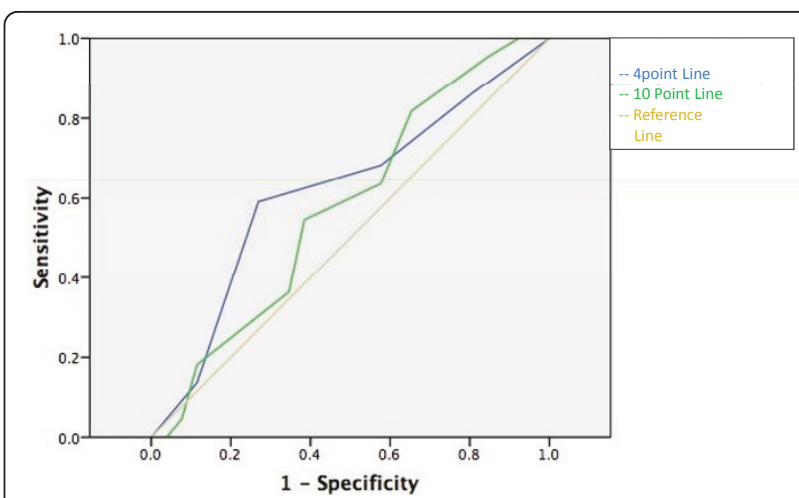

Figure 3 Gorelick 4- and 10-point scale predicting moderate $(\geq 5 \%)$ body weight change.

stable weight after $24 \mathrm{~h}$ in the hospital, and nearly all children achieved a stable weight by $72 \mathrm{~h}$. Since all patients in our study spent at least $24 \mathrm{~h}$ in the hospital, and $88 \%$ spent more than 3 days, it is likely that they had the opportunity to achieve a stable rehydration weight, so we believe that percent weight change with rehydration can be used as a valid gold standard for dehydration in our study.

While percent weight change with rehydration makes an excellent gold standard for the severity of dehydration, it is not a useful tool in practice, since it is not available at the time of presentation when a decision about how best to manage a child with diarrhea must be made. For many years, experts have recommended the use of physical exam signs to predict the severity of dehydration in children with diarrhea. Steiner et al. found in a systematic review that the most useful individual signs for predicting 5\% dehydration were abnormal capillary refill time, abnormal respiratory pattern, and abnormal skin turgor, which had positive likelihood ratios spanning from 2.0-4.1 [9]. However, none of these signs had very good negative likelihood ratios, meaning

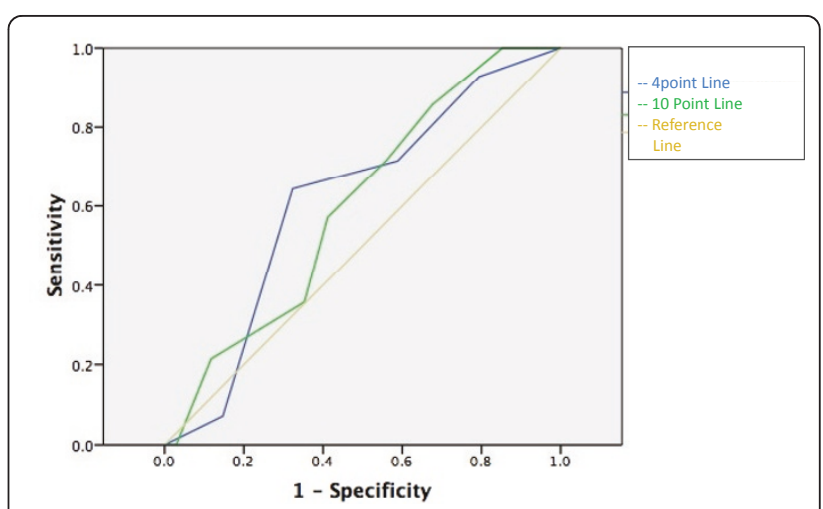

Figure 4 Gorelick 4- and 10-point scale predicting severe $(\geq 10 \%)$ body weight change. 


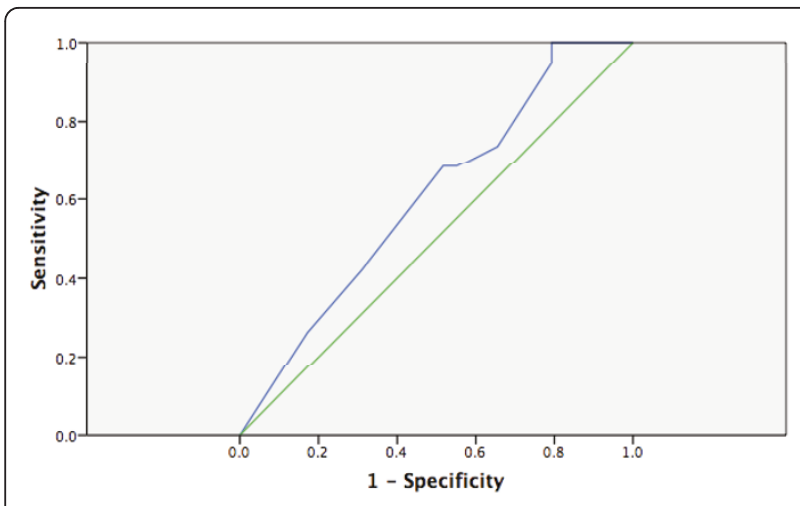

Figure 5 CDS predicting moderate $(\geq 6 \%)$ body weight change

that they were not useful for excluding severe dehydration in children. Steiner et al. concluded that no individual clinical sign had adequate sensitivity and specificity for the prediction of dehydration. Other studies that have looked at laboratory values, such as BUN, anion gap, base deficit, bicarbonate concentration, and urine specific gravity, have generally not found them to be very good predictors of dehydration status, with only bicarbonate greater than 15 or $17 \mathrm{mEq} / \mathrm{L}$ useful for reducing the likelihood of dehydration [6,13-16].

Given the limitations of individual clinical signs, several prior authors have tried to combine physical findings into clinical scales to predict percent dehydration in children. Gorelick created a 4-point and 10-point scale for assessing dehydration in children 1-60 months old presenting to Children's Hospital of Philadelphia, resulting in sensitivities of $79 \%$ and $87 \%$ and specificities of $82 \%$ and $85 \%$, respectively, for predicting $\geq 5 \%$ dehydration. The two scales had sensitivities of $82 \%$ and $90 \%$ and specificities of $83 \%$ and $90 \%$, respectively, for $\geq 10 \%$ dehydration. The CDS was derived at the Toronto Hospital for Sick Children and then prospectively validated at that site by specialized pediatric staff. In the validation study, Parkin et al. demonstrated likelihood ratios for moderate dehydration of 2.2, 1.3, and 5.2 for CDS scores of 0, 1-4, and $5-8$, respectively $[11,17]$.

Neither the Gorelick scale nor the CDS performed as well in our population of children in Rwanda as they did in North America. In fact, both scales had areas under the ROC curves statistically indistinguishable from the reference line, meaning they were no better than chance at predicting moderate or severe dehydration. In addition, the WHO scale, considered the standard of care in most low- and middle-income countries, although it has never been prospectively validated for predicting severe dehydration, also performed poorly in our population of children. To our knowledge, this study is the first to prospectively assess a clinical dehydration scale in a lowincome country, where children tend to present with more severe dehydration and be evaluated by personnel with less specialized training than their high-income country counterparts. Our study highlights the need for more research into better methods for detecting the severity of dehydration in children with diarrhea in resource-limited settings and supports a general rule that clinical scales derived in a high-income country setting require validation in resource-limited settings before being recommended for widespread use in these settings.

\section{Limitations}

Study subjects were a convenience sample based on investigator availability; overall, we enrolled about half of eligible patients. Our sample size was small, but powered to detect a negative likelihood ratio less than 0.1 and a positive likelihood ratio greater than 2. A priori, we had decided that for a clinical scale to be useful, it had to reduce the likelihood of severe dehydration by at least a factor of 10 when negative (so as not to miss any children who truly need IV fluids) while at least doubling the likelihood of severe dehydration when positive (so as to not to result in the wasted resources and adverse events that come from over-treating children with IV fluids who do not actually have severe dehydration). Essential data were missing for about $10 \%$ of enrolled patients, who were therefore excluded from analysis. In addition, only children admitted to the hospital were enrolled in our study in order to be able to obtain both pre- and post-rehydration weights for the purpose of determining the gold standard of percent weight change with rehydration. Therefore, the children enrolled in our study were likely more ill than the average child with diarrhea and/or vomiting in a low-income country setting, limiting the generizability of our results. We attempted to include only children who would have weight change based on rehydration by excluding those children presenting with severe malnutrition who would receive dietary supplementation. It is unlikely that children not receiving dietary supplementation would have gained weight from improved nutrition because $87 \%$ of children in our study spent less than 1 week in the hospital, so it is unlikely that they would have been able to gain a significant amount of protein-energy weight in that time period while being fed a standard Rwandan diet.

\section{Conclusion}

In this study, we found that the WHO scale, Gorelick scale, and CDS, when performed by general practice physicians and nurses in a resource-limited setting, were not accurate predictors of severe dehydration in children with diarrhea and/or vomiting. Due to the high prevalence and significant morbidity associated with diarrhea in children throughout the world, further research is necessary to develop and validate new clinical scales or 
other diagnostic tools with greater accuracy for assessing dehydration in children in resource-limited settings.

\section{List of abbreviations}

CDS: clinical dehydration scale; ROC curve: receiver operating characteristic curve; LR: likelihood ratio; WHO: World Health Organization.

\section{Acknowledgements}

We would like to thank Partners in Health/Inshuti Mu Buzima and the Rwandan Ministry of Health for their generous contributions to this study. Grant funding was provided by the Harvard Affiliated Emergency Medicine Residency Maryanne Povinelli Award.

\section{Author details}

'Department of Emergency Medicine, Brown University Alpert Medical School, Providence, RI, USA ${ }^{2}$ Rwinkwavu District Hospital, Eastern Province, Rwanda ${ }^{3}$ Kirehe District Hospital, Eastern Province, Rwanda ${ }^{4}$ Butaro District Hospital, Northern Province, Rwanda ${ }^{5}$ Partners in Health/Inshuti Mu Buzima, Rwanda ${ }^{6}$ Rhode Island Hospital, 593 Eddy Street, Claverick 274, Providence, RI 02904, USA

\section{Authors' contributions}

KP analyzed data and wrote the manuscript. SPS developed the study concept and design, and participated in the acquisition of data. IU, RBMM, $J M D, K S, J M$, and PN participated in data acquisition. SS provided administrative, technical, and material support. AL conceived the study, participated in its coordination, and edited the manuscript.

\section{Competing interests}

The authors declare that they have no competing interests.

Received: 1 June 2011 Accepted: 9 September 2011

Published: 9 September 2011

\section{References}

1. Boschi-Pinto C, Velebit L, Shibuya K: Estimating child mortality due to diarrhoea in developing countries. Bulletin of the World Health Organization; 2008:86:710-717.

2. Kosek M, Bern C, Guerrant R: The global burden of diarrhoeal disease, as estimated from studies published between 1992 and 2000. Bulletin of the World Health Organization; 2003:81:197-204.

3. World Health Organization: The treatment of diarrhoea: A manual for physicians and other senior health workers.[http://whqlibdoc.who.int/ publications/2005/9241593180.pdf], Date Accessed: July 3, 2011.

4. Center for Disease Control and Prevention: Viral Gastroenteritis.[http:// www.cdc.gov/ncidod/dvrd/revb/gastro/faq.htm], Date Accessed: July 3, 2011.

5. American Academy of Pediatrics Bullletin: Practice Parameter: The Management of Acute Gastroenteritis in Young Children. Pediatrics 1996, 3:424-435

6. Colletti JE, Brown KM, Sharieff GQ, Barata IA, Ishimine P: The management of children with gastroenteritis and dehydration in the emergency department. The Journal of Emergency Medicine 2010, 38:686-698.

7. United Nations International Children's Emergency Fund: New formulation of Oral Rehydration Salts (ORS) with reduced osmolarity.[http://www. supply.unicef.dk/catalogue/bulletin9.htm], Date Accessed: July 3, 2011.

8. King CK, Glass R, Bresee JS, Duggan C: Managing acute gastroenteritis among children: oral rehydration, maintenance, and nutritional therapy. MMWR Recomm Rep 2003, 52:1-16.

9. Steiner M, Darren D, Byerley J: Is This Child Dehydrated. JAMA 2004, 22:2746-2754, 291:

10. Fonseca BK, Holdgate A, Craig J: Enteral vs Intravenous Rehydration Therapy for Children With Gastroenteritis: A Meta-analysis of Randomized Controlled Trials. Arch Pediatric Adolesc Med 2004, 158:483-490.

11. Parkin P, Macarthur C, Khambalia A, Friedman J: Clinical and Laboratory Assessment of Dehydration Severity in Children with Acute Gastroenteritis. Clinical Pediatrics 2010, 3:235-239.
12. Gorelick M, Shaw K, Murphy K: Validity and Reliability of Clinical Signs in the Diagnosis of Dehydration in Children. Pediatrics 1997, 5:e1-6.

13. Mackenzie A, Barnes G, Shann F: Clinical signs of dehydation in childen. Lancet 1989, 2:605-607.

14. Yilmaz K, Karaboculoglu M, Citak A, Uzel N: Evaluation of laboratory tests in dehydrationed children with acute gastroenteritis. J Paediatr Child Health 2002, 38:226-228.

15. Teach SJ, Yates EW, Feld LG: Laboratory predictors of fluid deficit in acutely dehydrated children. Clinical Pediatr 1997, 36:395-400.

16. Vega RM, Avner J: A prospective study of the usefulness of clinical and laboratory parameters for prediction percentage of dehydration in children. Pediatr Emerg Care 1997, 13:179-182.

17. Friedman JN, Goldman RD, Srivastava R, Parking PC: Development of a clinical dehydration scale for use in children between 1 and 36 months of age. J Pediatr 2004, 145:201-207.

doi:10.1186/1865-1380-4-58

Cite this article as: Pringle et al:: Comparing the accuracy of the three popular clinical dehydration scales in children with diarrhea. International Journal of Emergency Medicine 2011 4:58.

\section{Submit your manuscript to a SpringerOpen ${ }^{\mathcal{O}}$ journal and benefit from:}

- Convenient online submission

- Rigorous peer review

- Immediate publication on acceptance

- Open access: articles freely available online

- High visibility within the field

- Retaining the copyright to your article

Submit your next manuscript at $\gg$ springeropen.com 